\title{
Calvarial and skull base metastases: expanding the clinical utility of Gamma Knife surgery
}

\author{
Clinical article
}

\author{
Rupesh Kotecha, M.D., ${ }^{1}$ Lilyana Angelov, M.D. ${ }^{2-4}$ Gene H. Barnett, M.D., M.B.A., ${ }^{2-4}$ \\ Chandana A. Reddy M.S., ${ }^{1}$ John H. Suh, M.D., ${ }^{1,3,4}$ Erin S. Murphy, M.D., ${ }^{1,3,4}$ \\ Gennady Neyman, Ph.D., ${ }^{1}$ and Samuel T. Chao, M.D. ${ }^{1,3,4}$
}

Departments of ${ }^{1}$ Radiation Oncology and ${ }^{2}$ Neurosurgery, ${ }^{3}$ Taussig Cancer Institute, and ${ }^{4}$ Brain Tumor and Neuro-Oncology Center, Cleveland Clinic Foundation, Cleveland, Ohio

\begin{abstract}
Object. Traditionally, the treatment of choice for patients with metastases to the calvaria or skull base has been conventional radiation therapy. Because patients with systemic malignancies are also at risk for intracranial metastases, the utility of Gamma Knife surgery (GKS) for these patients has been explored to reduce excess radiation exposure to the perilesional brain parenchyma. The purpose of this study was to report the efficacy of GKS for the treatment of calvarial metastases and skull base lesions.

Methods. The authors performed a retrospective chart review of 21 patients with at least 1 calvarial or skull base metastatic lesion treated with GKS during 2001-2013. For 7 calvarial lesions, a novel technique, in which a bolus was placed over the treatment site, was used. For determination of local control or disease progression, radiation therapy data were examined and posttreatment MR images and oncology records were reviewed. Survival times from the date of procedure were estimated by using Kaplan-Meier analyses.

Results. The median patient age at treatment was 57 years (range 29-84 years). A total of 19 (90\%) patients received treatment for single lesions, 1 patient received treatment for 3 lesions, and 1 patient received treatment for 4 lesions. The most common primary tumor was breast cancer ( $24 \%$ of patients). Per lesion, the median clinical and radiographic follow-up times were 10.3 months (range 0-71.9 months) and 7.1 months (range 0-61.3 months), respectively. Of the 26 lesions analyzed, $14(54 \%)$ were located in calvarial bones and $12(46 \%)$ were located in the skull base. The median lesion volume was $5.3 \mathrm{~cm}^{3}\left(\right.$ range $\left.0.3-55.6 \mathrm{~cm}^{3}\right)$, and the median prescription margin dose was 15 Gy (range 13-24 Gy). The median overall survival time for all patients was 35.9 months, and the 1-year local control rate was $88.9 \%$ (95\% CI 74.4\%-100\%). Local control rates did not differ between lesions treated with the bolus technique and those treated with traditional methods or between calvarial lesions and skull base lesions $(p>0.05)$. Of the 3 patients for whom local treatment failed, 1 patient received no further treatment and 2 patients responded to salvage chemotherapy. Subsequent brain parenchymal metastases developed in 2 patients, who then underwent GKS

Conclusions. GKS is an effective treatment modality for patients with metastases to the calvarial bones or skull base. For patients with superficial calvarial lesions, a novel approach with bolus application resulted in excellent rates of local control. GKS provides an effective therapeutic alternative to conventional radiation therapy and should be considered for patients at risk for calvarial metastases and brain parenchymal metastases.

(http://thejns.org/doi/abs/10.3171/2014.7.GKS141272)
\end{abstract}

\section{KeY Words • Gamma Knife surgery • skull • calvarial • metastasis • stereotactic radiosurgery}

ALVARIAL metastases are found in $15 \%-25 \%$ of all cancer patients, most often in those with systemic bony metastatic disease. Metastasis occurs via hematogenous spread, retrograde seeding through Batson's venous plexus, or direct extension through cranial foramina. ${ }^{1}$ Because progressive disease can lead to worsened neurological deficits or skeletal complications, thereby substantially compromising a patient's quality of life, local control of metastases in the calvaria and skull base is

Abbreviations used in this paper: GKS = Gamma Knife surgery; RTOG $=$ Radiation Therapy Oncology Group. critical. However, treatment of metastatic lesions in the calvarial bones and skull base, unlike other areas such as the extremities, is unique given the proximity to the brain parenchyma.

Treatment options for patients with calvarial and skull base metastases include resection for rapidly enlarging or solitary lesions, hormonal therapy for hormoneresponsive tumors, and chemotherapy. In addition, conventional external-beam radiation therapy is one of the mainstay treatment modalities for patients with intracranial metastases; approximately $70 \%$ of patients receive radiation therapy alone or in combination with surgery 
or chemotherapy. ${ }^{13}$ As an alternative to conventional external-beam radiation therapy, Gamma Knife surgery (GKS) is a safe, minimally invasive, and effective treatment that is increasingly being used to treat intracranial metastatic tumors. ${ }^{16}$ It provides good local control for brain parenchymal metastases, especially for those with radioresistant histologies, while significantly reducing the incidence of delayed neurotoxicity. ${ }^{16}$

Patients with metastasis to the calvaria or skull base are also at risk for brain parenchymal metastasis or additional metastatic lesions in other areas of the skull base and commonly undergo multiple radiation therapy treatments in closely overlapping regions, thereby complicating radiation therapy field matching or field avoidance. For these patients, given the poor effectiveness and substantial neurotoxicity associated with reirradiation for brain metastases, conventional external-beam radiation re-treatment options are limited?

Our objective with the study reported here was to describe an alternative to conventional external-beam radiation therapy. We report the clinical outcomes of patients who underwent GKS as primary treatment of lesions localized in the calvaria or skull base. We also describe a novel bolus-based treatment technique that we developed to treat superficial lesions in the calvarial bones.

\section{Methods}

We reviewed databases from the Cleveland Clinic Burkhardt Brain Tumor and Neuro-Oncology Center and Department of Radiation Oncology and identified 21 patients who had undergone GKS for calvarial and skull base metastases during 2001-2013. The metastatic lesions were classified by their originating site: calvaria or skull base. Lesions in the frontal, parietal, temporal, or occipital bones were classified as calvarial lesions. Lesions in the orbital plates of the frontal bone, greater or lesser wings of the sphenoid bone, basisphenoid, and basiocciput were classified as skull base lesions.

All patients in this study underwent Gamma Knife (Elekta AB) treatment as outpatients. Before 2007, a 201-source ${ }^{60} \mathrm{Co}$ Gamma Knife system was used; after that date, a 192-source unit (Perfexion) was used. A known limitation of the Gamma Knife planning system is limited dose calculation accuracy for sites within 5 $\mathrm{mm}$ of the surface because the software does not account for buildup at the scalp (Leksell Gamma Plan Reference Manual version 10, Elekta AB). Therefore, for 5 patients who received treatment for a total of 7 superficial calvarial lesions, a bolus skin-equivalent layer of at least 5 $\mathrm{mm}$ was positioned over the treatment site to artificially extend the surface to target distance and overcome this limitation. The bolus skin-equivalent layer was covered with a tightly fitted flexible silicone skin cap (Fig. 1A-D). All patients received a local anesthetic agent and an intravenous sedative/anxiolytic medication. A rigid Leksell stereotactic coordinate frame was applied to the patient's head, and high-resolution (1-mm slice) contrastenhanced MR images and CT images were acquired. For all patients, an MR-CT image match was performed to reduce any potential inaccuracies from image distortion.
The dose prescribed for the peripheral margin was typically based on the lesion size according to the Radiation Therapy Oncology Group (RTOG) $90-05$ protocol. ${ }^{19} \mathrm{Al}-$ though the maximum point dose to the overlying scalp was recorded in the treatment record, no modifications or constraints were placed on this point dose or the dose per surface area.

Clinical patient information was retrospectively obtained and included age, performance status (Karnofsky Performance Scale score), neurologic function score, sex, histologic type of primary tumor, presenting symptoms, treatment technique, local control, and overall survival. Radiation therapy data were abstracted and included the dose delivered to the periphery of the tumor volume, maximum dose, heterogeneity index, conformality index, and gradient index. The standard follow-up schedule consisted of appointments after completion of radiation therapy, typically at 3-month intervals. Follow-up examinations consisted of assessment of any new neurological or systemic symptoms, neurological physical examination, treatment-related toxicity assessment, and MR imaging. The presence or absence of intracranial metastases at time of treatment of the calvarial lesion or their occurrence during the follow-up period was also recorded. For patients for whom initial treatment failed, data regarding the use of salvage treatments, if any, were also recorded. Treatment-related toxicities were scored according to the National Cancer Institute Common Terminology Criteria for Adverse Events toxicity scale, version 3. When necessary, the Social Security Death Index was used to verify dates of death. Approval from the Cleveland Clinic Institutional Review Board was obtained before the study began.

Actuarial likelihood of local control and survival estimates were calculated according to the product-limit estimate (Kaplan-Meier) method. Multivariate analyses were calculated by using the Cox proportional hazards method. Data were collected in a Microsoft Excel database, and data analysis was performed by using SAS version 9.2 (SAS Institute). The threshold of statistical significance for differences was set at $\mathrm{p}<0.05$.

\section{Results}

The clinical features of the 21 patients in this study are presented in Table 1. Median patient age was 57 years (range 29-84 years), and median Karnofsky Performance Scale scores and neurological function scores were 80 (range 50-90) and 1 (range 0-3), respectively. The most common type of primary tumor was breast cancer (24\%). Most patients (57\%) were not receiving systemic medical therapy when skeletal metastases occurred. At the time of diagnosis of the calvarial or skull base lesion, concurrent diagnoses based on a review of systemic imaging studies were made for extracranial metastasis for $13(62 \%)$ patients and for brain parenchymal metastasis for $2(10 \%)$ patients. Patients with lesions in the skull base most often exhibited a constellation of symptoms including headaches ( 9 patients), localized pain or pressure (6 patients), cranial nerve dysfunction (3 patients), gait imbalance (1 patient), and/or invasion through the base of the skull (4 

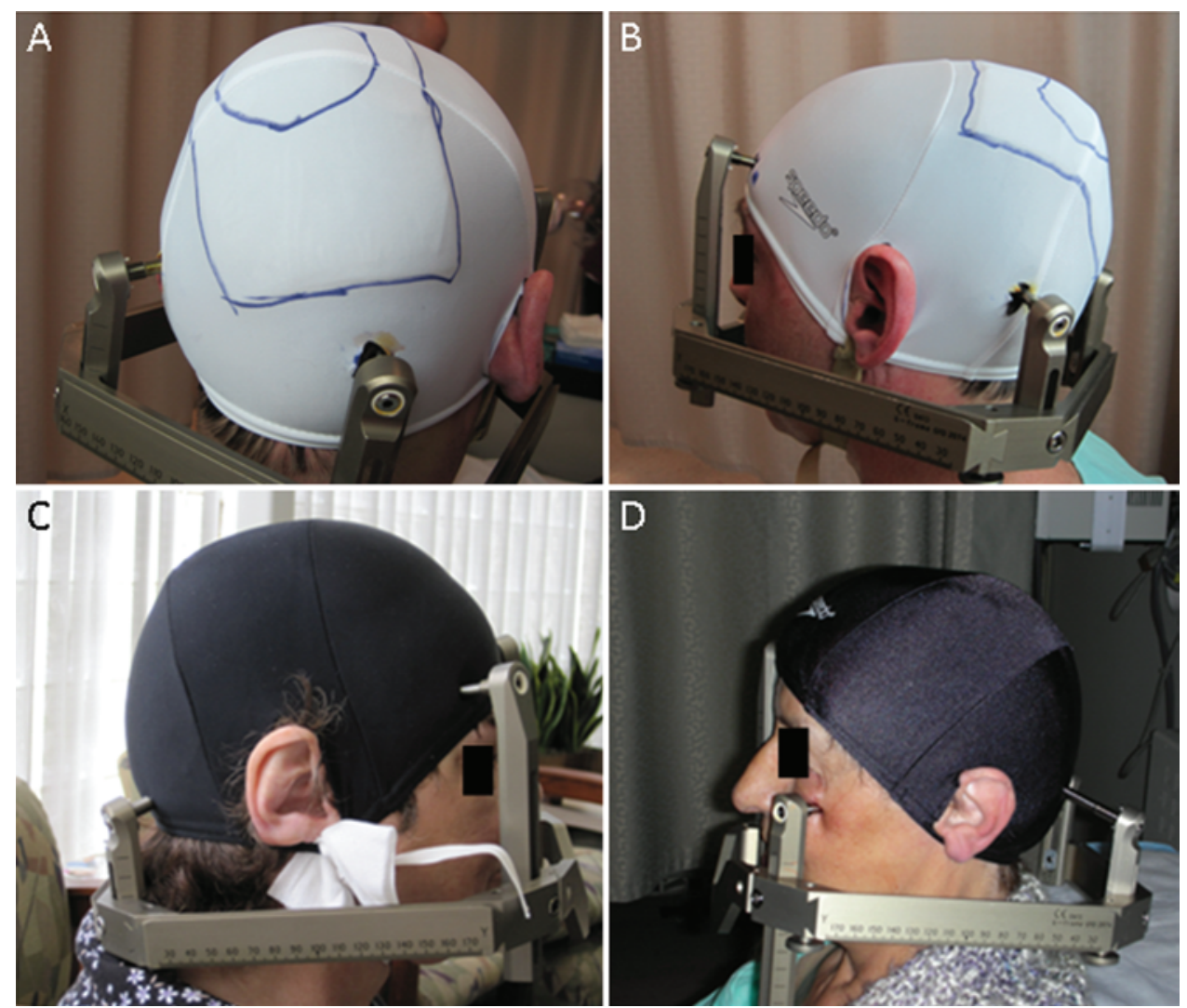

FIG. 1. Photographs of 2 patients who underwent treatment with the bolus-based radiosurgical technique developed for targeting superficial calvarial lesions. Both patients had undergone placement of a bolus skin-equivalent layer over the treatment site, which was then covered with a tightly fitted flexible silicone skin cap. The location of the underlying bolus skin-equivalent layer and the region of the target are outlined on the skin cap by blue marker in $\mathbf{A}$ and $\mathbf{B}$.

patients). Among the 5 patients with calvarial lesions, 1 received treatment for headaches and the other 4 received treatment for intracranial extension of disease into a sinus or through adjacent dural tissue.

A total of $14(67 \%)$ patients had not previously received any radiation therapy in close proximity to the calvarial or skull base metastasis. One patient had undergone GKS to a different site, and 6 (29\%) had previously received external-beam radiation therapy to an overlapping region of the newly diagnosed calvarial or skull base lesion.

The median time from initial diagnosis to development of symptomatic metastatic calvarial disease was 52.7 months (range 1.9-339.4 months). No patient received a diagnosis of skull metastasis at the time of initial cancer diagnosis. Most (90\%) patients underwent treatment for a single lesion; the maximum number of lesions treated was 4 (1 patient). The median lesion volume was $5.3 \mathrm{~cm}^{3}$ (range $0.276-55.6 \mathrm{~cm}^{3}$ ). A total of 26 lesions in 21 patients were treated; 14 (54\%) of these lesions were located in the calvarial bones, and $12(46 \%)$ were located in the skull base. The median prescription dose was 15 Gy to the $51 \%$ isodose line (range 13-24 Gy). The median conformality, heterogeneity, and gradient indexes were 1.67 (range 1.26-3.35), 1.95 (range 1.15-2.04), and 2.94 (range 2.23-3.53), respectively. The novel bolus-based technique was used to treat 7 lesions in 5 patients (Table
2). For patients who underwent treatment with the bolusbased technique, the maximum point (voxel) doses to the scalp were estimated $1 \mathrm{~mm}$ below the border of the bolus and ranged from 1550 to $2200 \mathrm{cGy}$. An example of a GKS treatment plan for using the bolus-based technique in a patient with a calvarial lesion is provided in Fig. 2.

Per lesion, the median clinical and radiographic follow-up times were 10.3 months (range 0-71.9 months) and 7.1 months (range 0-61.3 months), respectively. At the time of last follow-up visit, repeated neuroimaging indicated that local disease control had been achieved for $16(76 \%)$ patients who received treatment at $21(81 \%)$ sites. The 12-month local control rate for all lesions was $88.9 \%$ (95\% CI $74.4 \%-100 \%$ ). Rates of local control of lesions in the calvarial bones did not differ from those in the skull base $(p=0.3127)$. Also, rates of local control for lesions treated with traditional GKS and those treated by use of the novel bolus-based approach did not differ ( $\mathrm{p}=$ $0.6868)$.

Disease progressed locally for $3(14 \%)$ patients at a median of 10.7 months from the date of GKS, and 2 patients were lost to follow-up after procedure completion. Of note, none of the patients underwent whole-brain radiation therapy subsequent to GKS. Subsequent brain parenchymal metastases developed at a median of 15.4 months for 2 patients; both underwent GKS as subsequent treatment. Of the 3 patients who experienced local treat- 
TABLE 1: Patient characteristics

\begin{tabular}{lr}
\hline \multicolumn{1}{c}{ Characteristic } & No. $(\%)$ \\
\hline age $(\mathrm{yrs})$ & \\
$\geq 65$ & $7(33)$ \\
$<65$ & $14(67)$ \\
sex & \\
$\mathrm{M}$ & $12(57)$ \\
$\mathrm{F}$ & $9(43)$ \\
primary cancer & \\
breast adenocarcinoma & $5(24)$ \\
renal cell carcinoma & $3(14)$ \\
hemangiopericytoma & $3(14)$ \\
hepatocellular carcinoma & $2(10)$ \\
squamous cell carcinoma (head/neck) & $2(10)$ \\
other & $6(28)$ \\
extracranial metastasis & \\
yes & $13(62)$ \\
no & $8(38)$ \\
Karnofsky Performance Scale score & $13(71)$ \\
$\geq 80$ & $6(29)$ \\
$\leq 70$ & $12(57)$ \\
lesion site & \\
calvarial bone & \\
skull base & \\
neurological symptoms at diagnosis & \\
yes & \\
no & \\
\hline
\end{tabular}

ment failure, 1 remained symptom-free after initial GKS despite an increase in radiographic size of the metastatic lesion (this patient received supportive care only), and the other 2 patients received systemic chemotherapy for both local and distant progression of their disease. Two patients who received treatment for skull base lesions (primarily for headaches) were not classified as experiencing local failure according to radiographic criteria, but they continued to experience symptoms despite GKS. One of these patients, however, did experience a reduction in symptom frequency. The patient with headaches and lo- calized pain who received treatment for calvarial metastasis experienced significant improvement in pain relief and at the last follow-up visit was only intermittently experiencing headaches. For patients with lesion-associated pain, symptoms were stable or improved for all patients at the time of the last follow-up visit. Of the 3 patients with diplopia resulting from skull base involvement or cavernous sinus invasion, 2 experienced no worsening of their pretreatment cranial nerve dysfunction and 1 experienced mild improvement of diplopia but a persistent neurological deficit.

The median overall survival time was 35.9 months, and the 12-month survival rate was $76.5 \%$ (95\% CI $56.3 \%-96.6 \%)$. Survival rates did not differ between patients with calvarial or skull base lesions or between patients who did or did not undergo the bolus-based technique ( $p=0.3096$ and $p=0.7955$, respectively). None of the patients treated with the bolus-based technique experienced skin inflammation or scalp ulceration, although for 1 patient, a patch of alopecia developed over the treatment field. Grade 1 fatigue and confusion developed after the procedure for only 1 patient, and no radiosurgery-related deaths occurred among patients in this study group.

\section{Discussion}

This study describes the outcomes of patients treated with GKS targeting specific sites in the calvarial bones and skull base. On the basis of our experience treating 26 lesions in 21 patients, the 12-month rate of local control for all patients was approximately $90 \%$, indicating that GKS was an effective treatment modality. The use of GKS also enabled patients who had received prior external-beam radiation therapy to receive treatment to an overlapping region and concurrent and subsequent treatment of brain parenchymal metastasis. Our study also demonstrated that a bolus-based radiosurgery approach for treatment of superficial sites was feasible, safe, and effective.

The calvaria consists of an inner table, bone marrow space, and an outer table. Metastases to the calvarial bones usually involve all 3 skull layers. ${ }^{1}$ Although approximately half of patients with calvarial metastases are asymptomatic, presentation of patients with outer table, periosteal, or dural involvement may include localized pain in the setting of a palpable mass. ${ }^{1}$ Growth of the disease into the

TABLE 2: Treatment parameters for the 7 lesions treated with the bolus-based radiosurgery technique*

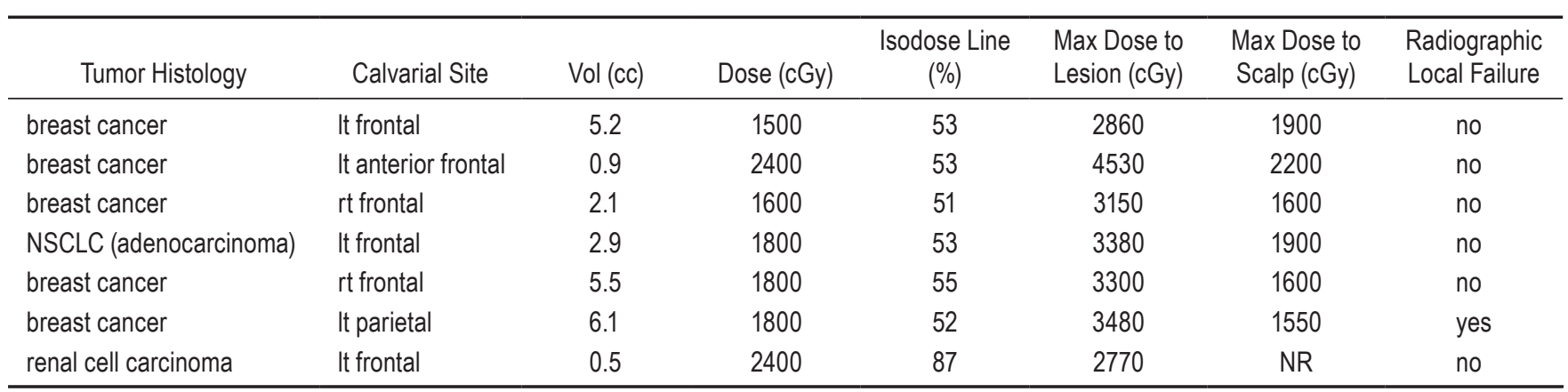

* Max = maximum; NR = data not retrievable; NSCLC = non-small cell lung carcinoma. 

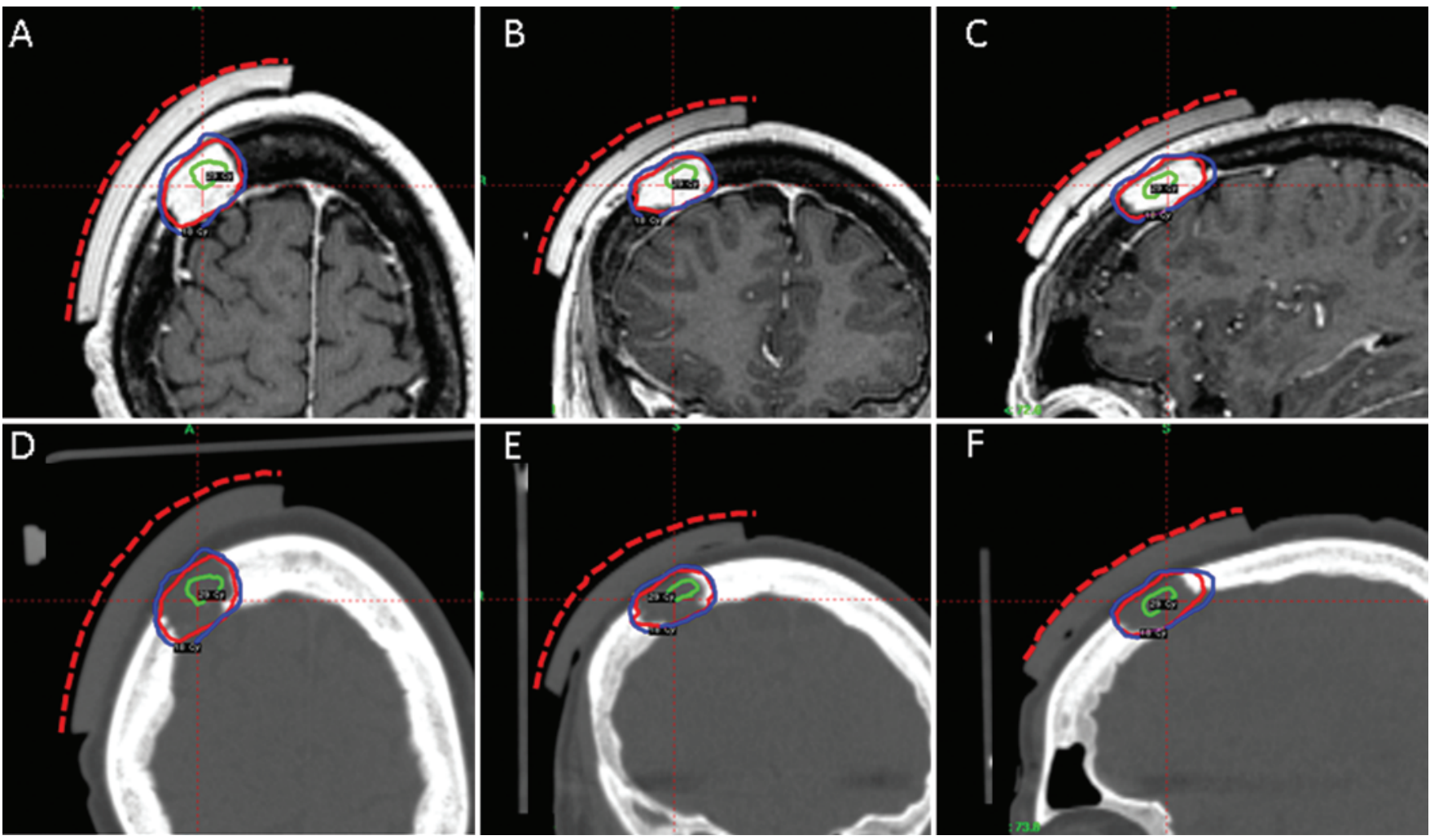

FIG. 2. GKS plan for a patient with a history of breast cancer and with a $1.8 \times 1.1-\mathrm{cm}$ contrast-enhanced osteolytic metastatic lesion in the right lateral frontal bone, extending through the inner and outer cortex of the bone and continuous with the dura. MR treatment planning images are shown in images A (axial), B (coronal), and C (sagittal); CT treatment planning images are shown in "bone windows" in $\mathbf{D}$ (axial), $\mathbf{E}$ (coronal), and $\mathbf{F}$ (sagittal). The lesion is outlined in red, and the 18-Gy prescription isodose line is outlined in blue. The green outline represents the 29-Gy isodose line. As seen in all images, a bolus skin-equivalent layer was positioned over the treatment site for this calvarial lesion.

inner table of the skull can result in neurological symptoms including headaches, neurological deficits, meningeal irritation, and seizures. ${ }^{1}$ Given this array of neurological sequelae, aggressive management is warranted for lesions invading the inner table of the bone and for those with dural involvement. In our study, most patients with calvarial lesions had involvement of the inner table of the skull and experienced uncontrolled headaches and referred pain despite medical therapy. To treat these superficial lesions, we developed a novel approach in which a bolus skin-equivalent layer was positioned over the site of treatment to extend the distance from the surface to the lesion enough to enable accurate dose calculation by the treatment planning software. Among patients who underwent treatment with this technique, the rate of local control was high; no patient experienced a significant adverse event. As an example, Fig. 3 shows representative treatment planning images of a patient with metastasis to the left paramedian frontal sinus extending through the inner table of the left anterior frontal calvaria. For this patient, follow-up MR images demonstrated complete resolution of the metastatic disease by 8 months (Fig. 4).

For treating superficial calvarial lesions, given the high surface dose and relatively limited dose distribution, megavoltage electron beams can also be used. Given the complex geometry of the curved skull, including anatomical distortion secondary to the metastatic lesion itself and the underlying brain parenchyma, compared with electrons, GKS provides a sharper dose falloff and limits the amount of adjacent tissue in the treatment field. Therefore, GKS allows for significant dose escalation as opposed to the traditional 8-Gy fraction size of conventional external-beam radiation therapy. Finally, because of the conformality of radiosurgical treatments, the use of GKS enables treatment of previous, concurrent, and subsequent calvarial metastases. An example is depicted in Figs. 5, 6, and 7 . Figure 5 shows images of a patient with a history of breast cancer metastasis to bone who received treatment with the bolus-based technique at 3 separate sites of calvarial disease in a single session. Each of the sites was treated independently with GKS with a sharp dose falloff. Alternatively, Figs. 6 and 7 show, respectively, planning with conventional external-beam radiation therapy with electron fields (doses are comparable to those used with GKS) and an 8-Gy fraction dose plan (more conventional dose). With en face electron fields, approximately $10 \%$ and $3 \%$ of the brain receives a dose of at least 8 Gy and $15 \%$ and $9 \%$ of the brain receives a dose of at least $4 \mathrm{~Gy}$ when the comparable GKS dosing (15-24 Gy) and the traditional 8-Gy dose, respectively, are used.

In addition to the treatment of simultaneous calvarial metastases, the use of GKS also enables treatment of concurrent and subsequent brain parenchymal metastases. In this study, 1 patient had been previously treated with 

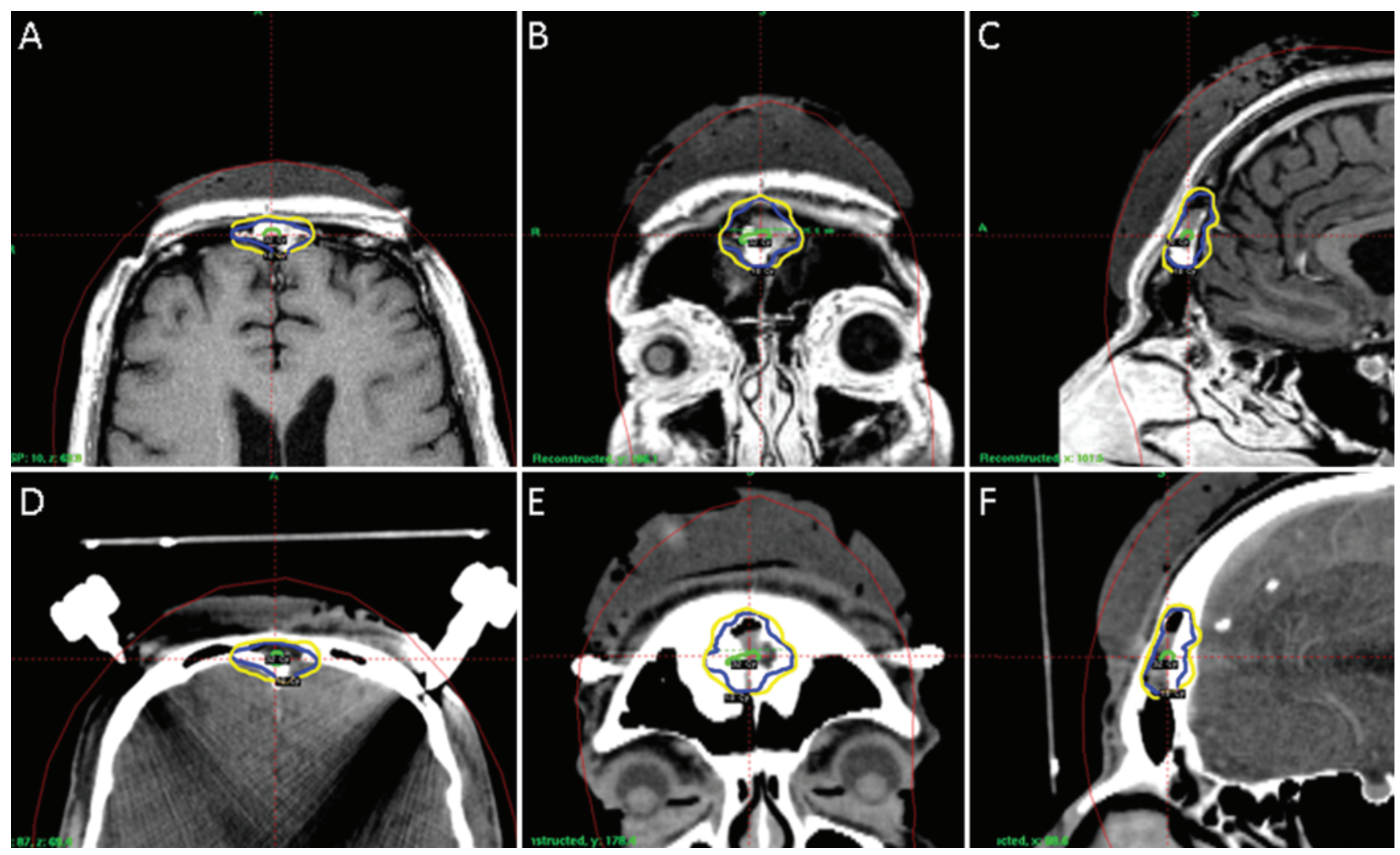

FIG. 3. GKS plan for a patient with a history of lung cancer and with metastasis to the left paramedian frontal sinus, measuring $12 \times 12 \times 7 \mathrm{~mm}$, extending through the inner table of the left anterior frontal calvaria. MR treatment planning images are shown in $\mathbf{A}$ (axial), $\mathbf{B}$ (coronal), and $\mathbf{C}$ (sagittal); CT treatment planning images are shown in $\mathbf{D}$ (axial), $\mathbf{E}$ (coronal), and $\mathbf{F}$ (sagittal). The lesion is outlined in blue, and the 18-Gy prescription isodose line is outlined in yellow. The green outline represents the 32-Gy isodose line. As seen in all images, a bolus skin-equivalent layer was positioned over the treatment site for this calvarial lesion.

GKS, 1 patient underwent concurrent treatment for brain parenchymal metastasis, and 1 patient underwent GKS for subsequent brain parenchymal metastasis. A fourth patient was treated with GKS for a concurrent brain parenchymal metastatic lesion and was found during followup to have an additional lesion, for which she again underwent GKS without complication. Given the high risk for additional calvarial or brain parenchymal lesions, lesions in the skull base are often treated with large-field radiation therapy or whole-brain radiation therapy. For example, in a series of 63 patients with metastatic skull tumors, $35(56 \%)$ patients had simultaneous brain parenchymal metastases and underwent whole-brain radiation therapy, whereas the other 28 (44\%) patients underwent local or whole-cranium radiation therapy. ${ }^{15}$ In this series, for $82 \%$ of patients with symptomatic skull metastases, symptoms either improved or remained stable. For patients with a limited number of lesions and good performance status, the white matter changes and neurotoxicity associated with whole-brain radiation therapy increased treatment complications at the cost of prophylactic and unnecessary treatment of the remaining brain parenchyma., 2,5

Among patients with metastases to the skull base, cranial nerve palsies were common. It is well known that in addition to headaches and localized pain, metastasis to the skull base can result in more serious neurological symptoms and cranial nerve deficits. For example, metastases involving the orbital bones can result in frontal pain, loss of sensation over the face, proptosis, diplopia, and decreased vision. ${ }^{6}$ These symptoms occur most frequently in the setting of metastatic prostate, lymphoma, and breast cancer..$^{13}$ Lesions in the parasellar region or cavernous sinus can result in frontal headaches, palsies of the oculomotor nerves, and loss of sensation over the face. ${ }^{6}$ Those in the middle cranial fossa can result in facial numbness, paresthesias, facial pain, and facial palsy. ${ }^{6}$ Lesions in the jugular foramen may result in a constellation of symptoms including hoarseness, dysphagia, and weakness of the sternocleidomastoid and/or trapezius muscle. ${ }^{6}$ If the hypoglossal nerve is involved, lesions in the occipital condyles can result in pain in the occipital distribution, neck stiffness, compression fracture, and unilateral tongue weakness. ${ }^{6}$

Given the multitude of neurological symptoms and clinical syndromes that can result from unrestricted growth of a metastatic lesion at the skull base, excellent control is necessary. In addition, because of the complex anatomy in this region, re-treatment of lesions in the skull base is difficult, and many physicians instead elect for combined modality approaches or larger treatment field sizes at initial presentation. Excellent local control has been demonstrated after use of GKS for these lesions. For example, a retrospective review of 71 University of Oklahoma patients who underwent both microsurgery and GKS for skull base tumors reported a reduction 

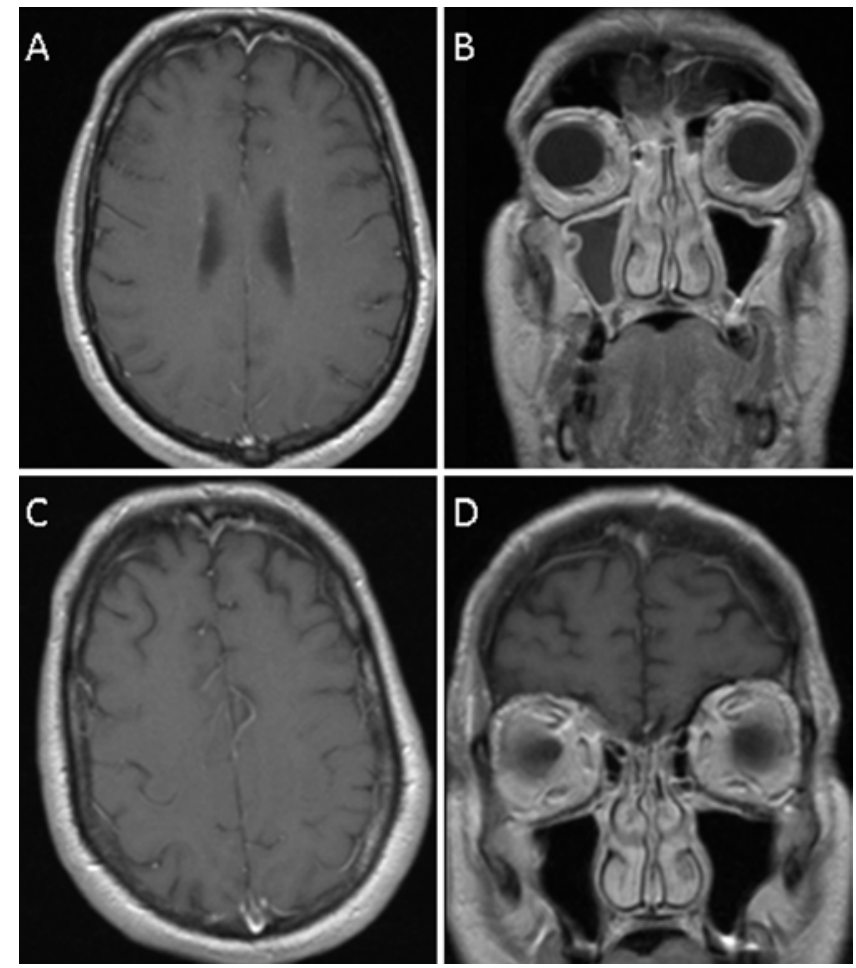

Fig. 4. Posttreatment MR images for a patient with a history of lung cancer and metastasis to the left paramedian frontal sinus, originally measuring $12 \times 12 \times 7 \mathrm{~mm}$, extending through the inner table of the left anterior frontal calvaria (see Fig. 3 for treatment planning images). The 2-month posttreatment MR images, A (axial) and B (coronal), demonstrate near-complete resolution of the extraaxial mass and mild residual enhancement measuring approximately $0.9 \times 0.7 \mathrm{~cm}$. The 8 -month posttreatment MR images, $\mathbf{C}$ (axial) and $\mathbf{D}$ (coronal), demonstrate complete resolution of the metastasis.

in tumor size for $34 \%$ of patients and an unchanged or smaller tumor size for $93 \%$ of patients. ${ }^{4}$ In the study reported here and as reported in the literature, GKS alone provides comparable rates of local radiographic control (Table 3), ${ }^{9,10,14,17}$ Furthermore, although not observed in this series, radiation therapy has the potential to improve cranial nerve function. ${ }^{18}$

For treatment of calvarial metastasis, the doses were based on the known safety profile of the guidelines determined by the RTOG $90-05 .{ }^{19}$ In general, systemic bone metastases are traditionally treated with low single-frac- tion doses based on the results of the RTOG 97-14 trial. The RTOG study showed, however, that rates of 3-year re-treatment were higher for patients who received a lower dose of $8 \mathrm{~Gy}$ in a single fraction than for patients who received a total dose of $30 \mathrm{~Gy}$ in 10 fractions $(15 \%$ vs $5 \%$, $\mathrm{p}=0.01) .{ }^{8}$ In our literature review, we found 1 case report of a patient with an occipital condyle metastasis treated with GKS, which resulted in a reduction in tumor size without evidence of disease recurrence after a prescribed peripheral marginal dose of $8 \mathrm{~Gy}$ (with a maximal dose of $17.77 \mathrm{~Gy}) .{ }^{12}$ In our study, however, destruction of both tables of the skull was typical and the underlying dura was often involved. Therefore, considering the adjacent brain parenchyma, we applied our traditional brain metastasis dosing guidelines to maximize tumor control.

Because of the complexities of re-treatment and the importance of tumor control in the skull base, typical conventional external-beam radiation therapy doses for skull base metastases range from $30 \mathrm{~Gy}$ to $36 \mathrm{~Gy}$, delivered at 3 Gy per fraction; improvement is expected for approximately $80 \%$ of patients. ${ }^{20}$ Moreover, there may be a doseresponse relationship, as suggested by a Memorial SloanKettering experience in which response rates for patients who received increasing doses of $24 \mathrm{~Gy}, 30 \mathrm{~Gy}$, and $36 \mathrm{~Gy}$ improved by $57 \%, 79 \%$, and $90 \%$, respectively. ${ }^{20}$ Therefore, for patients with a good long-term prognosis (controlled primary cancer, good performance status, limited concurrent medical conditions, and long life expectancy), higher doses up to 50 Gy are also reasonable. ${ }^{3}$ Of note, approximately $80 \%$ of patients who received radiation therapy have sustained improvement until their last follow-up visit or time of death..$^{20}$ In addition to dose escalation, the timing of treatment initiation might also affect effectiveness of treatment. In the institutional experience reported by Vikram and $\mathrm{Chu}$, improvement in symptoms was experienced by $87 \%$ of patients who received treatment within 1 month of symptom onset, by $69 \%$ who received treatment within $1-3$ months, and by only $25 \%$ of patients whose treatment was delayed more than 3 months. ${ }^{20}$ From these data, we can infer that earlier treatments and dose escalation might be associated with improved treatment response. Radiosurgery would extend this response further by possibly increasing tumor control in areas of the base of skull that would be difficult to re-treat while also enabling treatment of a metastatic lesion in close proximity to a previously treated site, either cranial or intracranial.

In this study, no patient who underwent treatment for

TABLE 3: Selected institutional studies of GKS for primary and secondary malignant tumors of the skull base*

\begin{tabular}{lccccccc}
\hline Author \& Year & $\begin{array}{c}\text { No. of } \\
\text { Patients }\end{array}$ & $\begin{array}{c}\text { Median } \\
\text { Follow-Up (mos) }\end{array}$ & $\begin{array}{c}\text { Median Tumor } \\
\text { Vol }\left(\mathrm{cm}^{3}\right)\end{array}$ & $\begin{array}{c}\text { Median Prescription } \\
\text { Margin Dose (Gy) }\end{array}$ & 1 Yr PFS (\%) & 1 Yr OS (\%) & Toxicity† \\
\hline Miller et al., 1997 & 32 & 27.6 & 14.6 & 15 & NR & 82.0 & optic neuropathy \\
Iwai et al., 2005 & 21 & 9.0 & 9.9 & 14 & 68.0 & 13 mos (MS) & none \\
Kano et al., 2009 & 37 & 12.9 & 6.3 & 14 & 77.6 & 36.6 & NR \\
Pan et al., 2013 & 43 & 14.0 & 7.2 & 14 & 89.0 & 74.0 & decreased vision \\
present study & 12 & 10.1 & 11.6 & 15 & 66.7 & 69.8 & confusion/fatigue \\
\hline
\end{tabular}

* MS = median survival; NR = not reported; OS = overall survival; PFS = progression-free survival.

$\dagger$ Toxicities, if any, occurred in 1 patient in each study. 

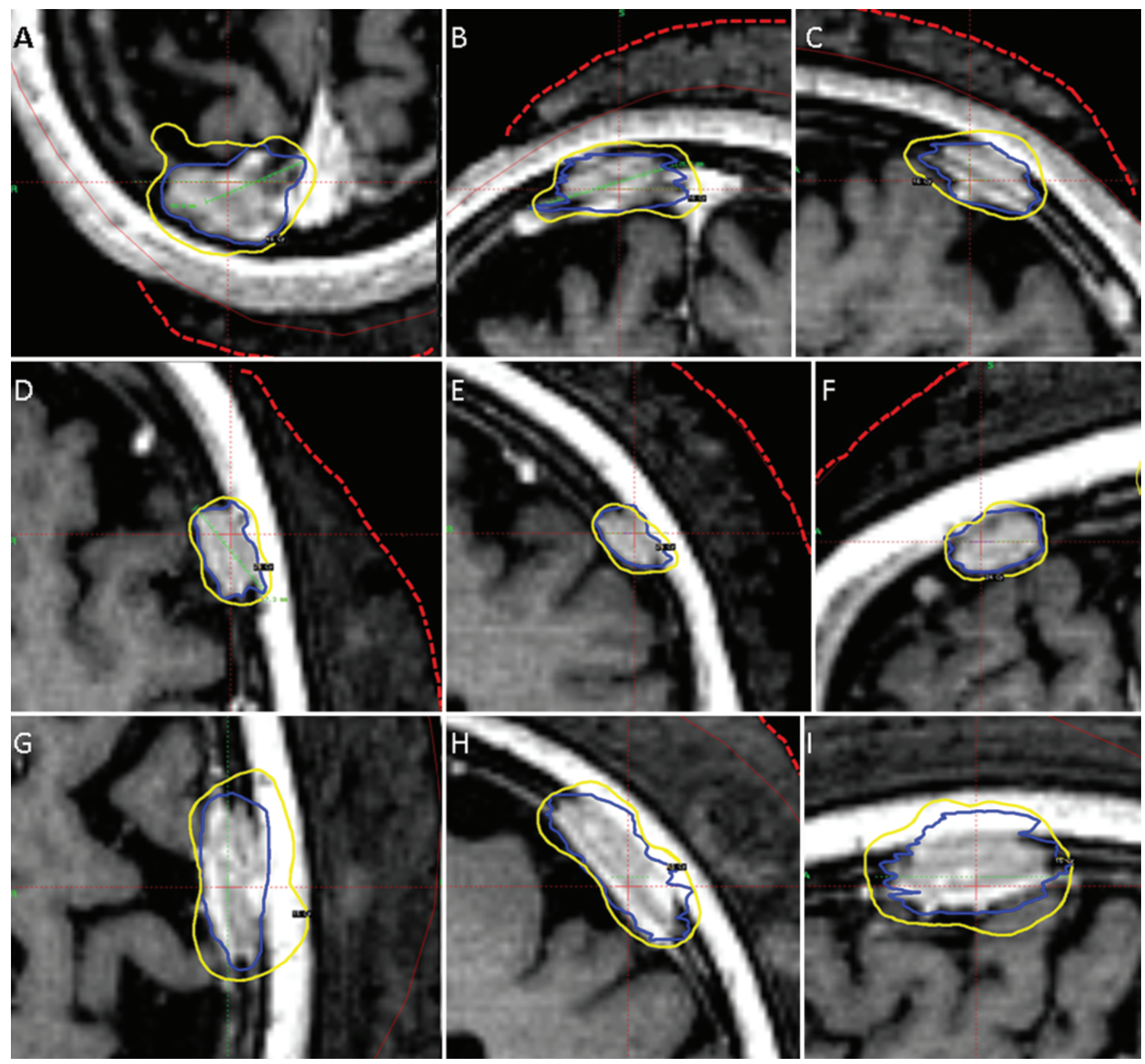

FIG. 5. GKS plans for a patient with a history of breast cancer and concurrent metastases (3 lesions) to the calvarial bones, extending through the inner table into the underlying dura. MR treatment planning images are shown for each lesion in axial (A, $\mathbf{D}$, and $\mathbf{G})$, coronal $(\mathbf{B}, \mathbf{E}$, and $\mathbf{H})$, and sagittal (C, F, and I) projections. The 3 lesions are outlined in blue, and the prescription isodose line is outlined in yellow $(16,24$, and $15 \mathrm{~Gy}$, respectively). The superficial border of the bolus skin-equivalent layer is outlined in red. Green indicates the ruler used to measure the size of the lesions.

calvarial lesions experienced worsening of local symptoms after radiosurgical treatment or significant complication as a result of this particular treatment technique. Specifically, no skin inflammation or scalp ulceration developed in any patient. Although no maximum dose constraints were placed on the overlying scalp at the time of treatment planning, the maximum voxel dose for all patients in this series was below the 26-Gy point dose $(<0.03 \mathrm{cc})$ recommended by the RTOG 0631 protocol, a Phase II/III trial of image-guided radiosurgery for localized spine metastasis. Moreover, although alopecia is an expected side effect of this procedure, for only 1 patient did a documented patch of alopecia develop over the treatment field. In addition to the previously mentioned complications, major treatment-related toxicities from GKS to these sites are expected to be quite rare and are probably limited to complications of cerebral edema or radiation-induced changes in the adjacent parenchymal tissue. In the study reported here, only 1 patient, who underwent treatment for skull base metastasis, experienced a neurological reaction (confusion and fatigue) to radiation therapy, which was managed with steroids. More se- 

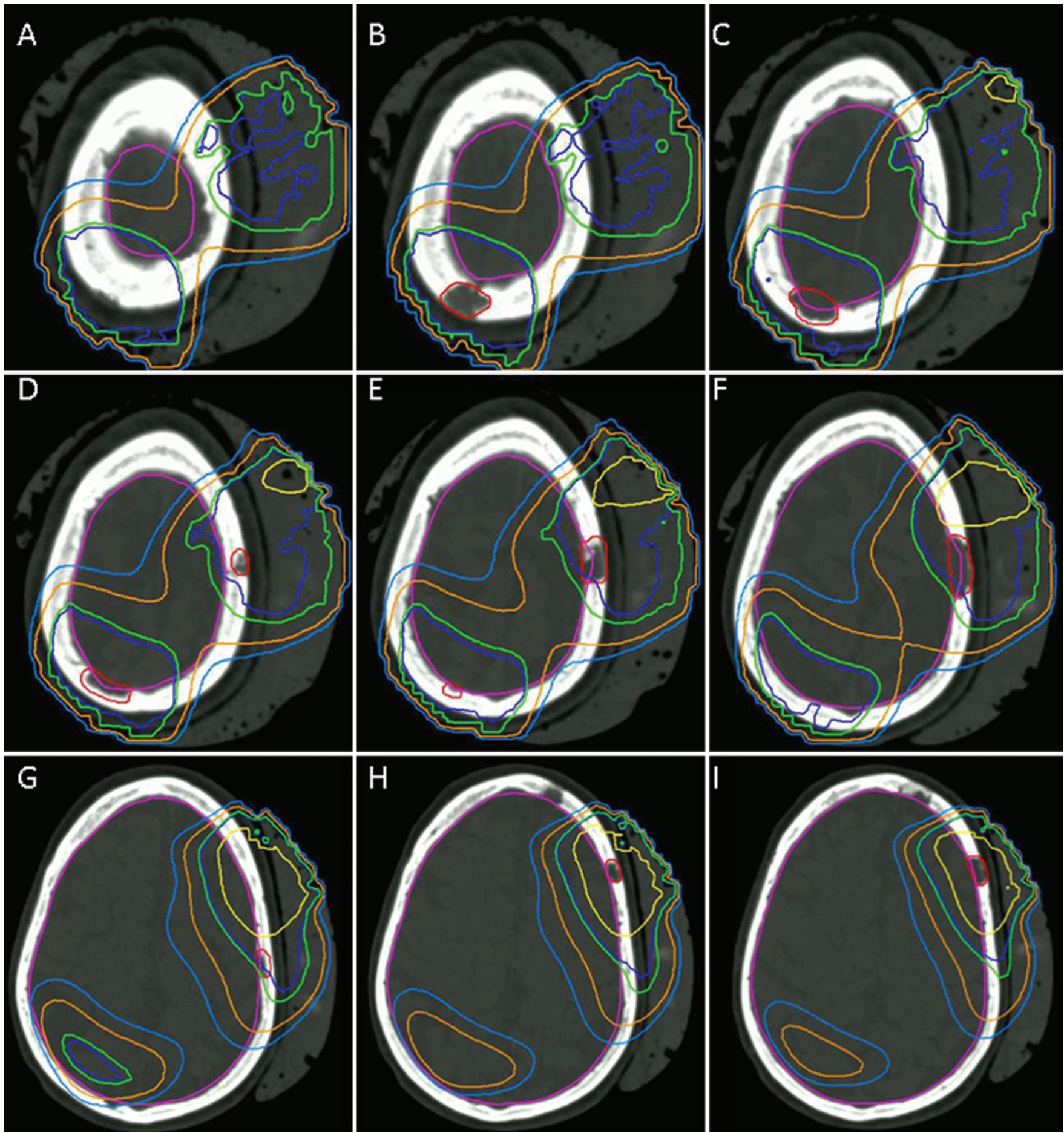

FIG. 6. Conventional external-beam radiation therapy treatment plan using en face electron fields for a patient with a history of breast cancer and with concurrent metastases ( 3 lesions) to the calvarial bones, extending through the inner table into the underlying dura (see Fig. 5 for comparison GKS plan). Axial CT images are oriented from superior to inferior (A-I). The 3 calvarial metastatic lesions are outlined in red, and the same doses used in GKS were prescribed (16, 24, and 15 Gy). The 24-Gy isodose line is in yellow, 16-Gy in dark blue, 15-Gy in green, 8-Gy in orange, and 4-Gy in light blue. A bolus skin-equivalent layer can be seen in all images. Please note that these are representative slices and that not all slices are included.

rious reactions have been reported in the literature: Kim et al. reported a case of cerebrospinal fluid leakage after radiosurgery for skull base metastasis from renal cell carcinoma; the leakage was successfully managed by endoscopic sinus surgery. ${ }^{11}$

\section{Conclusions}

The results of this study demonstrate that GKS can be used in selected patients with metastasis to the calvarial bones or skull base. GKS was an effective and safe 
R. Kotecha et al.

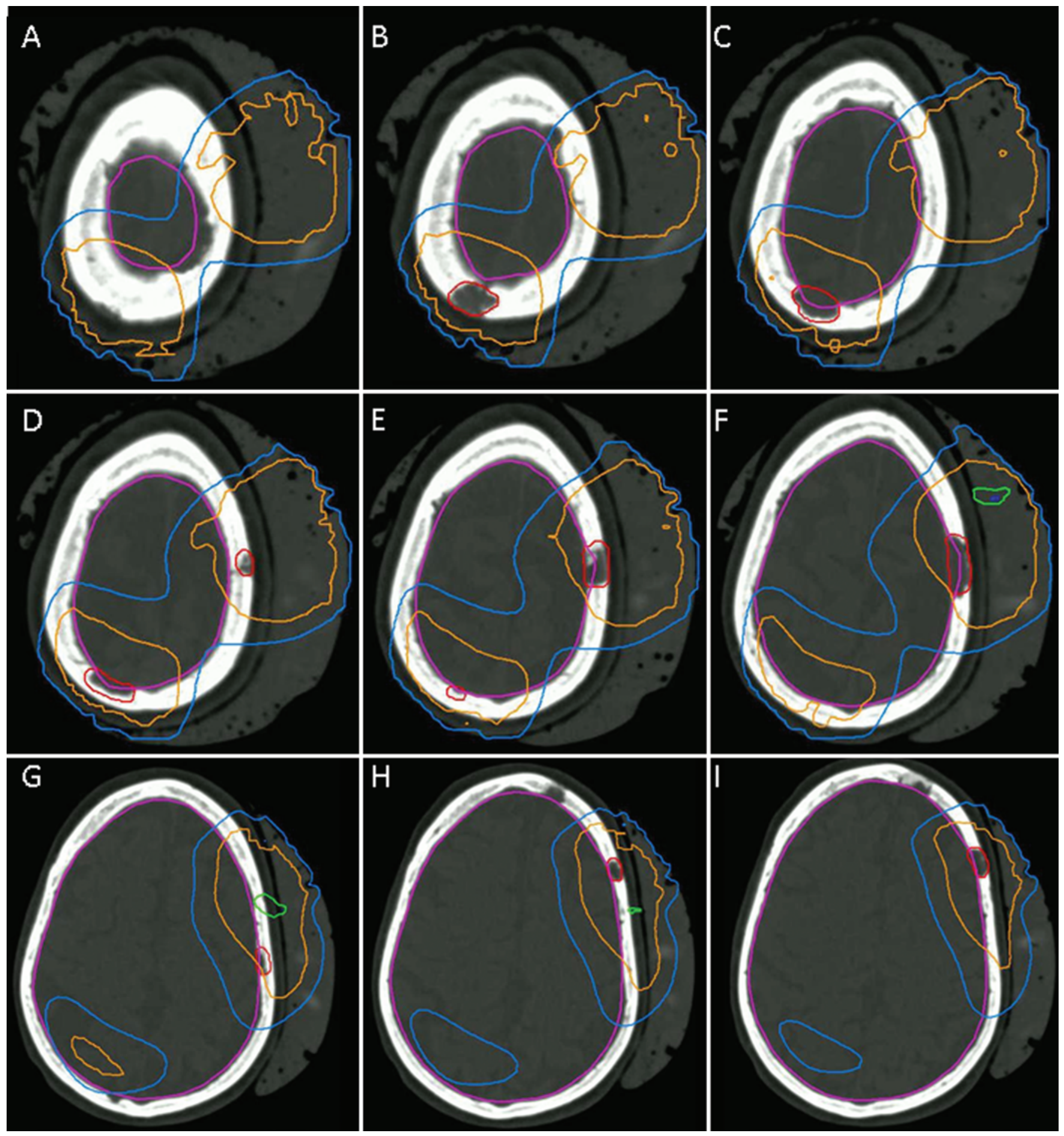

Fıg. 7. Conventional external-beam radiation therapy treatment plan using en face electron fields for a patient with a history of breast cancer and with concurrent metastases (3 lesions) to the calvarial bones, extending through the inner table into the underlying dura (Fig. 5 for comparison GKS plan). Axial CT images are oriented from superior to inferior (A-I). The brain is outlined in pink-purple. The 3 calvarial metastatic lesions are outlined in red, and a dose of 8 Gy was prescribed. The 15-Gy isodose line is in green, 8-Gy in orange, and 4-Gy in light blue. A bolus skin-equivalent layer can be seen in all images. Please note that these are representative slices and that not all slices are included.

treatment modality for patients who had received prior overlapping radiation therapy, for patients in whom concurrent treatment to other skeletal lesions could be performed with high conformality, and in patients who need- ed treatment for brain parenchymal metastasis (either concurrent or subsequent). For patients with superficial calvarial lesions, a novel approach with bolus application is safe, feasible, and effective and does not seem to subject 
patients to increased treatment-associated toxicity. Therefore, GKS provides an effective therapeutic alternative to conventional radiation therapy and should be considered for a subset of patients with calvarial metastases as well as brain parenchymal metastases.

\section{Acknowledgments}

The authors acknowledge Eric Murray for creating dosimetry plans for a comparative analysis between GKS and conventional external-beam radiation therapy and Amy Moore for her expertise with medical writing and editing.

\section{Disclosure}

This work had no specific funding source. Dr. Kotecha and Ms. Murphy report no conflict of interest concerning the materials or methods used in this study or the findings specified in this paper. Dr. Barnett has performed consulting and/or speaking services for Monteris Medical, Inc., and has received royalty payments from Mako Surgical Corp., Roche (Hoffman-La Roche, Ltd.). Dr. Suh has performed consulting services for Varian Medical Systems; Dr. Neyman has performed consulting and/or speaking services for Ele$\mathrm{kta} \mathrm{AB}$; and Dr. Chao is on the speaking bureau for Varian Medical Systems (honorarium).

Author contributions to the study and manuscript preparation include the following. Conception and design: Chao, Kotecha, Angelov, Neyman. Acquisition of data: Chao, Kotecha, Angelov, Neyman. Analysis and interpretation of data: Chao, Kotecha, Reddy. Drafting the article: Chao, Kotecha. Critically revising the article: all authors. Reviewed submitted version of manuscript: all authors. Approved the final version of the manuscript on behalf of all authors: Chao. Statistical analysis: Chao, Kotecha, Reddy. Study supervision: Chao.

\section{References}

1. Bradley WG (ed): Neurology in Clinical Practice: The Neurological Disorders. Philadelphia: Butterworth-Heinemann, 2004, p 1455

2. Chang EL, Wefel JS, Hess KR, Allen PK, Lang FF, Kornguth DG, et al: Neurocognition in patients with brain metastases treated with radiosurgery or radiosurgery plus whole-brain irradiation: a randomised controlled trial. Lancet Oncol 10: 1037-1044, 2009

3. Da Silva AN, Schiff D: Dural and skull base metastases. Cancer Treat Res 136:117-141, 2007

4. Francel PC, Bhattacharjee S, Tompkins P: Skull base approaches and gamma knife radiosurgery for multimodality treatment of skull base tumors. J Neurosurg 97 (5 Suppl): 674-676, 2002

5. Fujii O, Tsujino K, Soejima T, Yoden E, Ichimiya Y, Sugimura $\mathrm{K}$ : White matter changes on magnetic resonance imaging following whole-brain radiotherapy for brain metastases. Radiat Med 24:345-350, 2006

6. Greenberg HS, Deck MD, Vikram B, Chu FC, Posner JB: Metastasis to the base of the skull: clinical findings in 43 patients. Neurology 31:530-537, 1981

7. Hazuka MB, Kinzie JJ: Brain metastases: results and effects of re-irradiation. Int J Radiat Oncol Biol Phys 15:433-437, 1988

8. Howell DD, James JL, Hartsell WF, Suntharalingam M, Machtay M, Suh JH, et al: Single-fraction radiotherapy versus multifraction radiotherapy for palliation of painful vertebral bone metastases-equivalent efficacy, less toxicity, more convenient: a subset analysis of Radiation Therapy Oncology Group trial 97-14. Cancer 119:888-896, 2013

9. Iwai Y, Yamanaka K, Yoshimura M: Gamma knife radiosurgery for cavernous sinus metastases and invasion. Surg Neurol 64:406-410, 2005

10. Kano H, Niranjan A, Kondziolka D, Flickinger JC, Lunsford LD: The role of palliative radiosurgery when cancer invades the cavernous sinus. Int J Radiat Oncol Biol Phys 73:709715, 2009

11. Kim CH, Chung SK, Dhong HJ, Lee JI: Cerebrospinal fluid leakage after gamma knife radiosurgery for skull base metastasis from renal cell carcinoma: a case report. Laryngoscope 118:1925-1927, 2008

12. Krishnamurthy S, Navarro-Martín A, Maitz A: Gamma Knife radiosurgery for occipital condyle metastasis. Clin Transl Oncol 11:622-624, 2009

13. Laigle-Donadey F, Taillibert S, Martin-Duverneuil N, Hildebrand J, Delattre JY: Skull-base metastases. J Neurooncol 75:63-69, 2005

14. Miller RC, Foote RL, Coffey RJ, Gorman DA, Earle JD, Schomberg PJ, et al: The role of stereotactic radiosurgery in the treatment of malignant skull base tumors. Int J Radiat Oncol Biol Phys 39:977-981, 1997

15. Mitsuya K, Nakasu Y, Horiguchi S, Harada H, Nishimura T, Yuen S, et al: Metastatic skull tumors: MRI features and a new conventional classification. J Neurooncol 104:239-245, 2011

16. Monaco EA, Grandhi R, Niranjan A, Lunsford LD: The past, present and future of Gamma Knife radiosurgery for brain tumors: the Pittsburgh experience. Expert Rev Neurother 12:437-445, 2012

17. Pan J, Liu AL, Wang ZC: Gamma knife radiosurgery for skull base malignancies. Clin Neurol Neurosurg 115:44-48, 2013

18. Seymore $\mathrm{CH}$, Peeples WJ: Cranial nerve involvement with carcinoma of prostate. Urology 31:211-213, 1988

19. Shaw E, Scott C, Souhami L, Dinapoli R, Kline R, Loeffler J, et al: Single dose radiosurgical treatment of recurrent previously irradiated primary brain tumors and brain metastases: final report of RTOG protocol 90-05. Int J Radiat Oncol Biol Phys 47:291-298, 2000

20. Vikram B, Chu FC: Radiation therapy for metastases to the base of the skull. Radiology 130:465-468, 1979

Manuscript submitted June 9, 2014.

Accepted July 25, 2014.

This paper was presented in abstract form at the 17th International Leksell Gamma Knife Society Meeting, New York, New York, May 11-15, 2014.

Please include this information when citing this paper: DOI: 10.3171/2014.7.GKS 141272.

Address correspondence to: Samuel T. Chao, M.D., Department of Radiation Oncology, T28, 9500 Euclid Ave., Cleveland, OH 44195. email: chaos@ccf.org. 